\title{
Dclk1 Inhibition Cancels 5-FU-induced Cell-cycle Arrest and Decreases Cell Survival in Colorectal Cancer
}

\author{
YUKI SUEHIRO $^{1}$, YOSHIHIRO TAKEMOTO $^{1}$, ARATA NISHIMOTO ${ }^{2}$, KOJI UENO $^{1}$, BUNGO SHIRASAWA ${ }^{2}$, \\ TOSHIKI TANAKA ${ }^{1}$, NARUJI KUGIMIYA ${ }^{1}$, ATSUSHI SUGA ${ }^{1}$, EIJIRO HARADA ${ }^{1}$ and KIMIKAZU HAMANO ${ }^{1}$ \\ ${ }^{1}$ Department of Surgery and Clinical Science, ${ }^{2}$ Department of Medical Education, \\ Yamaguchi University Graduate School of Medicine, Ube, Japan
}

\begin{abstract}
Background/Aim: 5-Fluorouracil (5-FU) is frequently used in colorectal cancer treatment, but with limited success. The aim of the present study was to explore the cytotoxic effects of 5-FU, in combination with inhibition of doublecortin-like kinase 1 (Dclk1), a tumor stem cell marker that regulates pro-survival signaling in colorectal cancer cells, in the human colon cancer cell line, COLO-320. Materials and Methods: The effects of 5-FU treatment plus Dclk1 inhibition on the phosphorylation of checkpoint kinase 1 (Chk1), cell cycle, DNA damage, apoptosis, and cell survival in COLO-320 cells were evaluated. Results: Combined treatment with 5-FU and a Dclk1 inhibitor, LRRK2-IN-1 (LRRK), decreased 5-FU-induced phosphorylation of Chkl and canceled 5-FU-induced cell-cycle arrest at the $S$ phase. Combined treatment with 5-FU and LRRK failed to induce poly (ADP-ribose) polymerase 1 (PARP-1) cleavage, but tended to decrease cell survival compared to individual treatment with 5-FU or LRRK. Conclusion: These results indicate that a combination of 5-FU and LRRK may be an effective, novel approach for colorectal cancer therapy.
\end{abstract}

Colorectal cancer is the third leading cause of cancer-related death in the world and represents a severe threat to human health (1). 5-Fluorouracil (5-FU), a pyrimidine analogue, induces the inhibition of DNA synthesis through inhibition of thymidylate synthetase and is misincorporated into DNA

Correspondence to: Yoshihiro Takemoto, Department of Surgery and Clinical Science, Yamaguchi University Graduate School of Medicine, 1-1-1 Minami-Kogushi, Ube, Yamaguchi 755-8505, Japan. Tel: +81 836222261, Fax: +81 836222423, e-mail: ytake@yamaguchi-u.ac.jp and Arata Nishimoto, Department of Medical Education, Yamaguchi University Graduate School of Medicine, 1-1-1 Minami-Kogushi, Ube, Yamaguchi 755-8505, Japan. Tel: +81 836853450, Fax: +81 836853451, e-mail: anishimo@yamaguchi-u.ac.jp

Key Words: Colorectal cancer, 5-fluorouracil, LRRK2-IN-1, cytotoxicity, doublecortin-like kinase 1 , cell-cycle arrest, checkpoint kinase 1 phosphorylation. and RNA, leading to the induction of cell-cycle arrest at $\mathrm{G}_{1} / \mathrm{S}$ phase (2). Presently, the combination of infused 5$\mathrm{FU} /$ leucovorin (LV) plus irinotecan or oxaliplatin, known as the FOLFIRI (3) and FOLFOX4 regimens (4), represents the standard first-line chemotherapy. However, the emergence of tumor cells with acquired pharmacological resistance to these agents limits the antitumor effect of these therapies. Tumor cell-acquired pharmacological resistance leads to development of metastasis, which is responsible for approximately $90 \%$ of colorectal cancer-related mortality (5).

5-FU treatment induces the phosphorylation of checkpoint kinase 1 (Chk1), a cell-cycle checkpoint protein, and arrests cell-cycle progression at the $S$ phase $(6,7)$. Thus, combined treatment with 5-FU and Chk1 inhibition is expected to increase cell death due to lethal chromosomal instability caused by the progression of the cell cycle without repairing the damaged DNA at the arrested S phase. Therefore, the combined effect of 5-FU and Chk1 inhibition has previously been examined. As expected, Chk1 inhibition sensitized the cells to 5-FU-induced cytotoxicity (6-8). Furthermore, this combined treatment is found to increase cell death in colorectal cancer cells compared to individual treatment with 5-FU or Chk1 inhibitor (9). However, Chk1 plays an important role in the cell-cycle checkpoint of normal cells, and therefore combined treatment with 5-FU and Chk1 inhibitor may lead to severe side-effects caused by cytotoxicity in normal cells. Therefore, it is necessary to identify a molecular target specifically in cancer cells to increase the cytotoxic effect of 5-FU without causing severe side-effects.

Doublecortin-like kinase 1 (Dclk1) encodes a $\mathrm{Ca}^{2+} /$ calmodulin-dependent protein kinase-like domain and regulates microtubule polymerization (10). Dclk1 expression distinguishes the tumor cells from normal stem cells in the intestine (11) and regulates pro-survival signaling and selfrenewal in the intestinal tumor cells (12). Dclk1 is upregulated and associated with metastasis and prognosis in colorectal cancer (13). Dclk1 expression is also found in hyperplastic polyp and low-grade adenomas, and there is a tendency to increase with worsening severity of dysplasia 
(14). Moreover, small-molecule kinase inhibitor LRRK2-IN1 (LRRK) has been shown to suppress Dclk1 activity, thereby demonstrating potent anti-cancer activity including inhibition of cancer cell proliferation, migration, and invasion as well as induction of apoptosis and cell-cycle arrest (15). We recently reported that the combined treatment of pancreatic cancer cells with gemcitabine (GEM) and a Dclk1 inhibitor, LRRK, facilitated cell cycle progression without arrest at $\mathrm{S}$ phase, through the suppression of Chk1 phosphorylation (16). Furthermore, the cytotoxic effect of GEM was enhanced by Dclk1 inhibition in the pancreatic cancer cells (16). However, it remains unclear whether the cytotoxic effect of 5-FU can be enhanced by Dclk1 inhibition in colorectal cancer cells.

In this study, the aim was to explore the combined cytotoxic effect of 5-FU treatment and Dclk1 inhibition in the human colon cancer cell line, COLO-320. The effect of individual and combined treatment with 5-FU and LRRK on Chk1 phosphorylation, cell cycle, DNA damage, apoptosis, and cell survival was examined.

\section{Materials and Methods}

Cell line and cell culture. Human colon cancer cell line, COLO-320 was purchased from Riken BioResource Center (Tsukuba, Japan). COLO-320 cells were cultured in RPMI 1640 (Thermo Fisher Scientific Inc, Waltham, MA, USA) supplemented with $10 \%$ fetal bovine serum (Thermo Fisher Scientific Inc.). COLO-320 cells were maintained at $37^{\circ} \mathrm{C}$ in a humidified atmosphere containing $5 \% \mathrm{CO}_{2}$.

Reagents. 5-FU (99\% HPLC purified) was purchased from SigmaAldrich (St. Louis, MO, USA), dissolved in dimethyl sulfoxide (DMSO) and stored at $4^{\circ} \mathrm{C}$. LRRK2-IN-1 (99\% LCMS purified) was purchased from ChemScene (Monmouth Junction, New Jersey, USA), dissolved in DMSO and stored at $-80^{\circ} \mathrm{C}$.

Western blot analysis. COLO-320 cells were treated with vehicle (control), 5-FU $(10 \mu \mathrm{M})$, LRRK $(50 \mu \mathrm{M})$, or with a combination of 5 -FU $(10 \mu \mathrm{M})$ and LRRK $(50 \mu \mathrm{M})$ for $24 \mathrm{~h}$ or $48 \mathrm{~h}$. After harvesting, the cells were washed with phosphate buffered saline (PBS) and lysed with NP40 lysis buffer [50 mM Tris-HCl (pH 7.4), $150 \mathrm{mM}$ $\mathrm{NaCl}, 1 \% \mathrm{NP} 40$, and $1 \mathrm{x}$ protease inhibitor]. The lysed cells were incubated on ice for $30 \mathrm{~min}$. After centrifugation at $20,000 \times \mathrm{g}$ for $20 \mathrm{~min}$ at $4^{\circ} \mathrm{C}$, and the supernatants were collected as cell lysates. For western blot analysis, equivalent amounts of cell lysates $(30 \mu \mathrm{g})$ were mixed with 6x Sample Buffer Solution with Reducing Reagent (Nacalai Tesque, Kyoto, Japan) for sodium dodecyl sulfatepolyacrylamide gel electrophoresis (SDS-PAGE) and incubated at room temperature for $15 \mathrm{~min}$. Cell lysates mixed with sample buffer were separated by SDS-PAGE using the SuperSep Ace 10\% polyacrylamide gel (Wako, Osaka, Japan). The proteins separated by SDS-PAGE were transferred onto an Immobilon-P PVDF transfer membrane (Merck KGaA, Darmstadt, Germany), and the membrane was blocked with Tris-buffered saline with Tween-20 (TBST) containing $5 \% \mathrm{w} / \mathrm{v}$ bovine serum albumin for $1 \mathrm{~h}$ at room temperature. The membrane was immunoblotted with the specific primary antibody diluted at 1:1000 and incubated overnight at $4^{\circ} \mathrm{C}$.
The membrane was then washed with TBST and incubated with the specific secondary antibody diluted at 1:5000 for $1 \mathrm{~h}$ at room temperature. After washing with TBST, the immune complexes were visualized using the Amersham ECL Prime Western blotting detection reagent (GE Healthcare Life Sciences, Chicago, IL, USA). Band intensity was quantified using ImageJ software (National Institutes of Health, Bethesda, Maryland, USA). The primary antibodies used in this study were anti-DCAMKL1 antibody (Abgent, San Diego, CA, USA), anti-Phospo-Chk1 (S317) antibody (Cell Signaling Technology, Danvers, MA, USA), anti-Chk1 antibody (Abcam, Cambridge, UK), anti-PARP-1 (F-2) antibody (Santa Cruz Biotechnology, Santa Cruz, CA, USA), and anti- $\alpha-$ tubulin antibody (10D8) (Santa Cruz Biotechnology). The secondary antibodies used in this study were goat polyclonal anti-mouse and anti-rabbit immunoglobulins conjugated with horseradish peroxidase (HRP) (Dako, Glostrup, Denmark).

Fluorescence-activated cell sorting (FACS) and cell cycle analysis. To synchronize COLO-320 cells at $\mathrm{G}_{0} / \mathrm{G}_{1}$ phase of cell cycle, RPMI containing $10 \%$ FBS was substituted with serum-free medium on the following day. After serum starvation for $24 \mathrm{~h}$, serum-free RPMI was substituted with RPMI containing 10\% FBS. COLO-320 cells were treated with 5-FU $(10 \mu \mathrm{M})$, LRRK $(50 \mu \mathrm{M})$, or 5-FU $(10 \mu \mathrm{M})$ and LRRK $(50 \mu \mathrm{M})$ for $48 \mathrm{~h}$. Vehicle (DMSO)-treated cells served as control group. After treatment, the cells were harvested and resuspended in PBS. Then, the cells were separated by centrifugation at $140 \times g$ for $5 \mathrm{~min}$, fixed in cold $70 \%$ ethanol at $-20^{\circ} \mathrm{C}$ for $60 \mathrm{~min}$. The fixed cells were washed with Cell Staining Buffer (Biolegend, San Diego, CA, USA). Afterwards, cells were incubated with propidium iodide (PI)/RNase (Immunostep, Salamanca, Spain) and FITC anti-H2A.X Phospho (Ser139) antibody (Biolegend). After incubation, the cells were analyzed using a BD FACSCalibur (BD Biosciences, San Jose, CA, USA).

Cell survival assay. For cell survival assays, $1 \times 10^{4}$ COLO-320 cells/100 $\mu \mathrm{l} /$ well were seeded in 96-well culture plates. After incubation for $24 \mathrm{~h}, \mathrm{COLO}-320$ cells were treated with 5-FU $(2 \mu \mathrm{M})$, LRRK $(20 \mu \mathrm{M}), 5$-FU $(2 \mu \mathrm{M})$ and LRRK $(20 \mu \mathrm{M})$, or vehicle for 48 h. Cell Count Reagent SF (Nacalai Tesque) was added, and the cells were incubated for $4 \mathrm{~h}$. Then, absorbance at $450 \mathrm{~nm}$ was measured using an iMark microplate reader (Bio-Rad, Hercules, CA, USA). The absorbance value was normalized to that in control cells.

Statistical analysis. Statistical analysis was performed using JMP Pro 13.1.0 software (SAS Institute Inc., Cary, NC, USA). Tukey's test was performed to analyze the differences between multiple groups. The criterion for statistical significance was $p<0.05$.

\section{Results}

Combined treatment with 5-FU and LRRK significantly decreased 5-FU-induced phosphorylation of Chkl. To investigate whether the combined treatment with 5-FU and a Dclk1 inhibitor, LRRK, inhibits Chk1 phosphorylation induced by 5 -FU treatment, COLO-320 cells were treated with 5-FU, LRRK, 5-FU and LRRK, or vehicle for $24 \mathrm{~h}$, followed by western blotting. 5-FU treatment significantly induced Chk1 phosphorylation in COLO-320 cells compared to the control (Figure 1A and B). Combined treatment with 5-FU and 
A

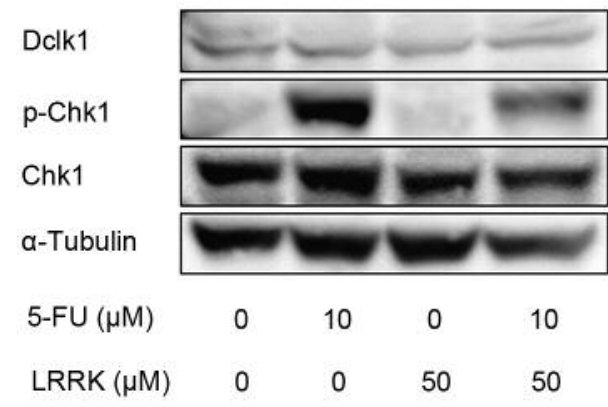

B

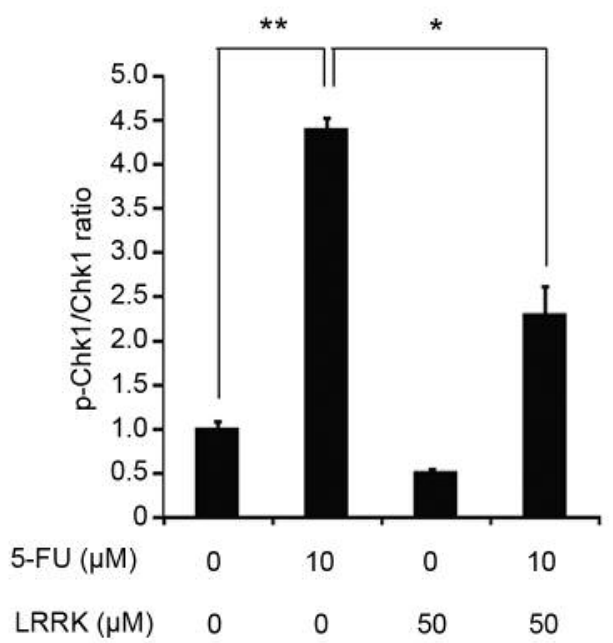

Figure 1. Effect of 5-FU, LRRK, or 5-FU and LRRK combined treatment on Chk1 phosphorylation. (A) Cell lysates were prepared from COLO-320 cells treated with vehicle (control), 5-FU $(10 \mu M)$, LRRK $(50 \mu M)$, or with a combination of 5-FU (10 $\mu M)$ and LRRK (50 $\mu M)$ for 24 h, and the expressions of Dclk1, phosphorylated Chk1 (p-Chk1), Chk1, and $\alpha$-Tubulin were detected by western blotting. $\alpha$-Tubulin was used as a loading control. (B) The intensity of each band was quantified using the ImageJ software. The ratio of p-Chk1 to Chk1 was normalized to that of control. Each bar represents the mean $\pm S D$ of three experiments. *p<0.05, **p<0.01. 5-FU, 5-Fluorouracil; LRRK, LRRK2-IN-1; Chk1, checkpoint kinase 1; p-Chk1, phosphorylated checkpoint kinase 1; Dclk1, doublecortin-like kinase 1.

LRRK significantly decreased 5-FU-induced phosphorylation of Chk1 compared to individual treatment with 5-FU (Figure $1 \mathrm{~A}$ and B). Dclk1 expression was detected in COLO-320 cells treated with 5-FU, LRRK, or with a combination of 5-FU and LRRK, and in control cells, as well (Figure 1A).

Combined treatment with 5-FU and LRRK abrogated 5-FUinduced cell-cycle arrest. To investigate the individual and combined effects of 5-FU and LRRK treatment on cell cycle progression, flow cytometry analyses were performed. COLO-320 cells were synchronized at $\mathrm{G}_{0} / \mathrm{G}_{1}$ phase after serum starvation for $24 \mathrm{~h}$. Then, serum-free medium was substituted with RPMI 10\% FBS medium containing vehicle (DMSO), 5-FU, LRRK, or 5-FU and LRRK. After incubation for $48 \mathrm{~h}$, cell cycle and DNA damage analyses were performed. Individual treatment with 5-FU induced cell-cycle arrest at the $S$ phase and individual treatment with LRRK increased the proportion of the cells at $\mathrm{G}_{2} / \mathrm{M}$ phase compared to the control (Figure 2, upper panel). Notably, combined treatment with 5-FU and LRRK canceled 5-FUinduced cell-cycle arrest at $\mathrm{S}$ phase (Figure 2, upper panel). Furthermore, the proportion of $\gamma-\mathrm{H} 2 \mathrm{~A}$ histone family member $X(\gamma-\mathrm{H} 2 \mathrm{AX})$-positive cells as a marker of DNA damage was investigated. The proportion of $\gamma-\mathrm{H} 2 \mathrm{AX}-$ positive cells was increased following individual treatment with 5-FU, but remained almost unchanged following individual treatment with LRRK compared to control cells
(Figure 2, lower panel). Unexpectedly, the proportion of $\gamma$-H2AX-positive cells was decreased following combined treatment with 5-FU and LRRK compared to individual treatment with 5-FU (Figure 2, lower panel).

Combined treatment with 5-FU and LRRK failed to induce poly (ADP-ribose) polymerase 1 (PARP-1) cleavage. To investigate the individual and combined effects of 5-FU and LRRK treatment on apoptosis, COLO-320 cells were treated with 5-FU, LRRK, 5-FU and LRRK, or vehicle for $48 \mathrm{~h}$, followed by western blotting analysis. Combined treatment with 5-FU and LRRK failed to induce PARP-1 cleavage when compared to individual treatment with 5-FU or LRRK. (Figures 3A and B).

Combined treatment with 5-FU and LRRK tended to decrease the survival of COLO-320 cells compared to individual treatment with 5-FU or LRRK. To investigate the individual and combined effects of 5-FU and LRRK treatment on cell survival, assays were performed using COLO-320 cells treated with vehicle, 5-FU, LRRK, or 5-FU and LRRK for $48 \mathrm{~h}$. The concentrations of 5-FU and LRRK ( $2 \mu \mathrm{M}$ and $20 \mu \mathrm{M}$, respectively) were determined based on the concentrations that induced $70-80 \%$ cell survival during individual treatment with 5-FU or LRRK. Cell survival was significantly decreased to a modest extent after individual treatment with 5-FU $(2 \mu \mathrm{M})$ or LRRK $(20 \mu \mathrm{M})$ compared to 

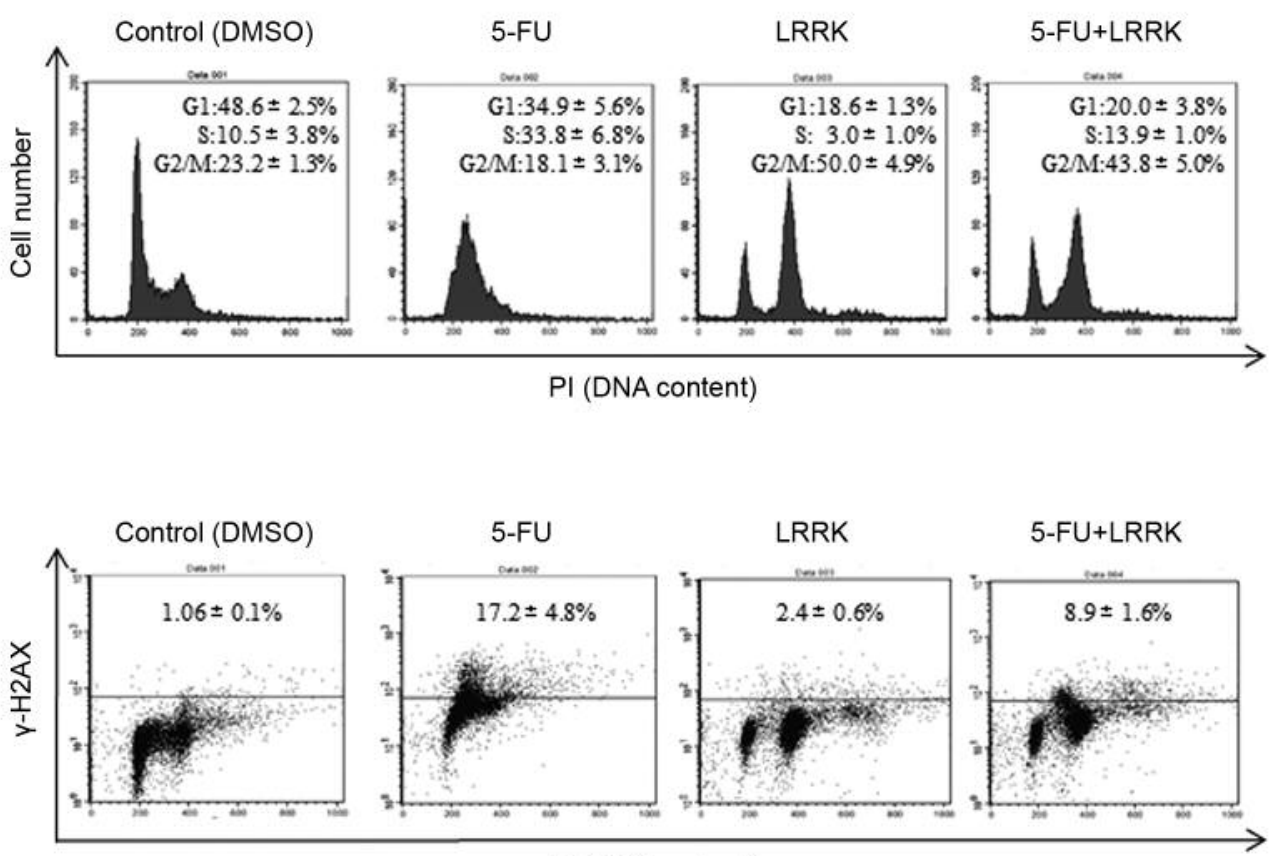

PI (DNA content)

Figure 2. Effect of 5-FU, LRRK treatment, or combined treatment with 5-FU and LRRK on cell cycle and DNA damage. After 24-h serum starvation, COLO-320 cells were treated with vehicle (DMSO; control), 5-FU (10 $\mu M)$, LRRK (50 $\mu M)$, or with a combination of 5-FU (10 $\mu M)$ and LRRK (50 $\mu M)$ for 48 h, followed by FACS analysis. The horizontal line shows the intensity of propidium iodide (PI) staining. The vertical line shows cell number (upper panel) and $\gamma$-H2AX expression (lower panel). $\gamma$-H2AX -positive cells were determined based on the criteria that the proportion of $\gamma$-H2AX-positive cells in COLO-320 cells treated with DMSO is approximately 1\%. The numbers in each upper panel and each lower panel indicate the cell proportion in each cell cycle phase and the proportion of $\gamma-H 2 A X$-positive cells, respectively. The values represent the mean $\pm S D$ of three experiments. 5-FU, 5-Fluorouracil; LRRK, LRRK2-IN-1; DMSO, dimethyl sulfoxide; FACS, fluorescence-activated cell sorting; $\gamma$-H2AX, $\gamma$-H2A histone family member X.

the control, and combined treatment with 5-FU and LRRK tended to decrease cell survival compared to individual treatment with 5-FU or LRRK (Figure 4).

\section{Discussion}

We recently reported that the cytotoxic effect of GEM is enhanced by Dclk1 inhibition, through the suppression of Chk1 phosphorylation in pancreatic cancer cells (16). Individual treatment with GEM was shown to induce cellcycle arrest at $\mathrm{S}$ phase through Chk1 phosphorylation and increase the proportion of $\gamma$-H2AX-positive cells. Individual treatment with LRRK also increased the proportion of $\gamma$-H2AX-positive cells, but allowed cell cycle progression. Notably, combined treatment with GEM and LRRK was shown to facilitate cell-cycle progression without arrest at $\mathrm{S}$ phase through the suppression of Chk1 phosphorylation and generate more $\gamma$-H2AX-positive cells compared to individual treatment with GEM or LRRK (16).

In this study, individual treatment of COLO-320 cells with 5 -FU induced cell-cycle arrest at $\mathrm{S}$ phase and generated $\gamma$-H2AX-positive cells to a modest extent. Individual treatment with LRRK increased the proportion of cells in $\mathrm{G}_{2} / \mathrm{M}$ phase and resulted in almost no $\gamma$-H2AX-positive cells. Combined treatment with 5-FU and LRRK allowed cell-cycle progression without arrest at $\mathrm{S}$ phase, whereas decreased $\gamma$-H2AX-positive cells when compared to individual treatment with 5-FU. The observed variations in the responses may be due to the different mechanisms of action of GEM and 5-FU. Upon cellular uptake, 5-FU is metabolized to FdUTP, FUTP, and FdUMP. The mechanism of action of each metabolic product is different. FdUTP inhibits DNA synthesis and is misincorporated into DNA (17). Similarly, FUTP inhibits RNA synthesis and is misincorporated into RNA $(18,19)$, while FdUMP inhibits thymidylate synthetase, leading to inhibition of DNA synthesis (20). On the other hand, GEM is a deoxycytidine analogue and is misincorporated into the DNA (21-23). Thus, among the 5-FU metabolic products, only FdUTP is misincorporated into DNA, while GEM is misincorporated into the DNA instead of cytidine. Therefore, it is beneficial to select an anti-cancer drug that induces more DNA damage, such as GEM, to enhance the combined effect of a DNA damaging reagent and LRRK. To confirm this 
A

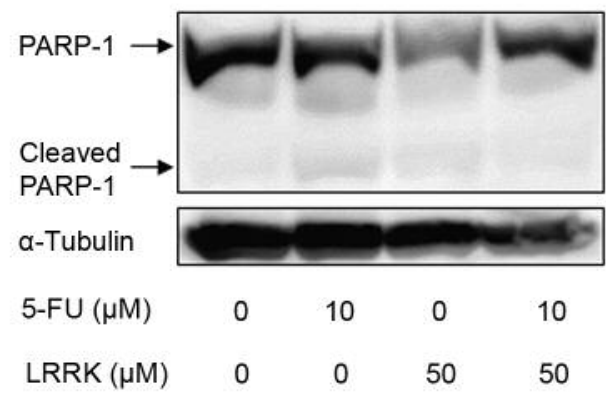

B

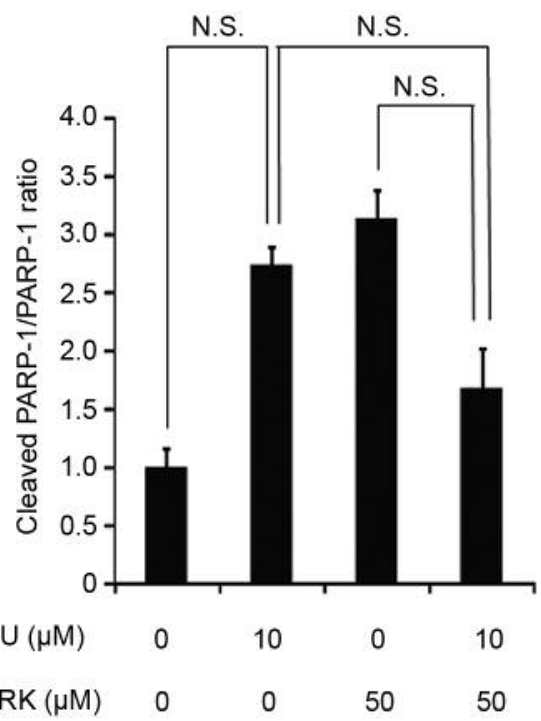

Figure 3. Effect of 5-FU, LRRK treatment, or combined treatment with 5-FU and LRRK on PARP-1 cleavage. (A) Cell lysates were prepared from COLO320 cells treated with vehicle (control), 5-FU (10 $\mathrm{MM}), \mathrm{LRRK}(50 \mu \mathrm{M})$, or with a combination of $5-F U(10 \mu M)$ and LRRK (50 $\mu M)$ for $48 \mathrm{~h}$, and the expression of PARP-1, cleaved PARP-1, and $\alpha$-Tubulin were detected by western blotting. $\alpha$-Tubulin was used as a loading control. (B) The intensity of each band was quantified using the ImageJ software. The ratio of cleaved PARP-1 to PARP-1 was normalized to that of control. Each bar represents the mean $\pm S D$ of three experiments. N.S., No significant difference. 5-FU, 5-Fluorouracil; LRRK, LRRK2-IN-1; PARP-1, poly (ADP-ribose) polymerase 1.

possibility, further research is required, to investigate whether cell survival rate in COLO-320 cells following combined treatment with GEM and LRRK is decreased compared to combined treatment with 5-FU and LRRK.

Furthermore, concerning apoptosis induction, it was demonstrated that individual treatment of COLO-320 cells either with LRRK ( $50 \mu \mathrm{M}$ for $48 \mathrm{~h}$ ) or with 5 -FU was not able to clearly induce PARP-1 cleavage. Similarly, combined treatment of COLO-320 cells with 5-FU and LRRK did not increase PARP-1 cleavage. In contrast, in our previous study on human pancreatic cancer MIA Paca2 cells, which were resistant to apoptosis induced by treatment with GEM, it was reported that PARP-1 cleavage was clearly induced both by individual treatment with LRRK (10 $\mu \mathrm{M}$ for $48 \mathrm{~h})$ and combined treatment with GEM and LRRK, as well (16). Thus, it could be hypothesized that COLO-320 cells were resistant to apoptosis induced by LRRK, while MIA Paca2 cells were not.

Combined treatment with 5-FU and LRRK failed to induce PARP-1 cleavage in COLO-320 cells, but tended to reduce cell survival, when compared to individual treatment with 5-FU or LRRK. We hypothesized that these results may be due to the different methods that were used, since western blotting was performed on cell lysates prepared from a fraction of the adherent cells in a well, while cell survival assay was performed on both the adherent and floating dead cells present in a well. To strengthen this hypothesis, apoptosis and cell survival should be analyzed by the same method using the same cell populations.

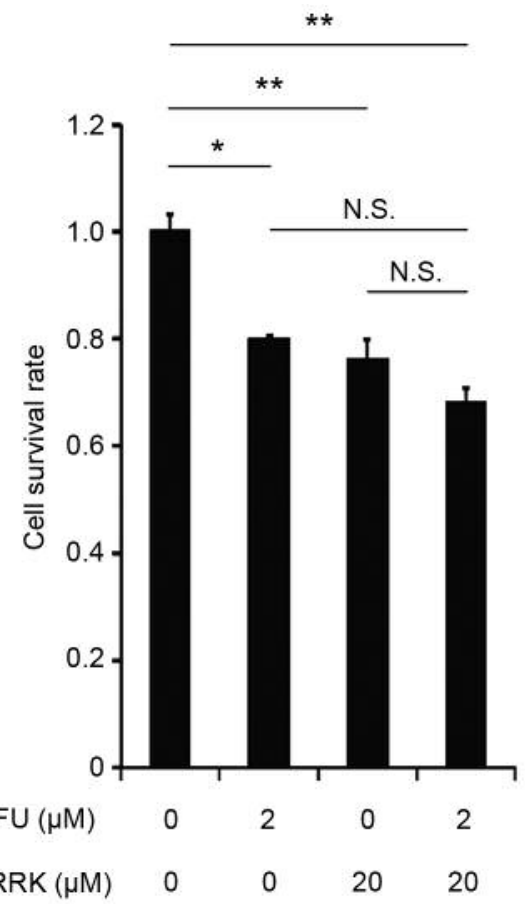

Figure 4. Effect of 5-FU, LRRK treatment, or combined treatment with 5-FU and LRRK on cell survival. COLO-320 cells were treated with vehicle (control), 5-FU $(2 \mu M)$, LRRK $(20 \mu M)$, or with a combination of 5-FU $(2 \mu M)$ and LRRK $(20 \mu M)$ for $48 \mathrm{~h}$, followed by cell survival assay. Cell survival rate was normalized to that in control cells. Each bar represents the mean $\pm S D$ of three experiments. $* p<0.05$, **p<0.01, N.S., no significant difference. 5-FU, 5-Fluorouracil; LRRK, LRRK2-IN-1. 
In conclusion, it was shown that the combined treatment of the human colorectal cancer cell line, COLO-320, with 5-FU and LRRK significantly decreased 5-FU-induced phosphorylation of Chk1 and canceled 5-FU-induced cellcycle arrest at the $\mathrm{S}$ phase, leading to a trend of decreased cell survival. Thus, our findings suggest that targeting Dclk1 in combination with $5-\mathrm{FU}$ is a potential future strategy for colorectal cancer treatment.

\section{Acknowledgements}

Te Authors would like to thank Ms. Yukari Hironaka for technical assistance in FACS and cell cycle analysis. They would also like to thank Editage (www.editage.jp) for English language editing. This study was supported by Grant-in-Aids for Young Scientific Research (B) (16K19932 to Y.T.) from the Japan Society for the Promotion of Science (JSPS).

\section{References}

1 Torre LA, Bray F, Siegel RL, Ferlay J, Lortet-Tieulent J and Jemal A: Global cancer statistics, 2012. CA Cancer J Clin 65: 87-108, 2015.

2 Johnson KR, Wang L, Miller MC, 3rd, Willingham MC and Fan W: 5-Fluorouracil interferes with paclitaxel cytotoxicity against human solid tumor cells. Clin Cancer Res 3: 1739-1745, 1997.

3 Douillard JY, Cunningham D, Roth AD, Navarro M, James RD, Karasek P, Jandik P, Iveson T, Carmichael J, Alakl M, Gruia G, Awad L and Rougier P: Irinotecan combined with fluorouracil compared with fluorouracil alone as first-line treatment for metastatic colorectal cancer: a multicentre randomised trial. Lancet 355: 1041-1047, 2000.

4 de Gramont A, Figer A, Seymour M, Homerin M, Hmissi A, Cassidy J, Boni C, Cortes-Funes H, Cervantes A, Freyer G, Papamichael D, Le Bail N, Louvet C, Hendler D, de Braud F, Wilson C, Morvan F and Bonetti A: Leucovorin and fluorouracil with or without oxaliplatin as first-line treatment in advanced colorectal cancer. J Clin Oncol 18: 2938-2947, 2000.

5 Christofori G: New signals from the invasive front. Nature 441: 444-450, 2006.

6 Xiao, Z, Xue, J, Sowin TJ, Rosenberg SH and Zhang H: A novel mechanism of checkpoint abrogation conferred by Chk 1 downregulation. Oncogene 24: 1403-1411, 2005.

7 Robinson HMR, Jones R, Walker M, Zachos G, Brown R, Cassidy J and Gillespie DAF: Chk1-dependent slowing of Sphase progression protects DT40 B-lymphoma cells against killing by the nucleoside analogue 5-fluorouracil. Oncogene 25: 5359-5369, 2006.

8 Fujinaka Y, Matsuoka K, Iimori M, Tuul M, Sakasai R, Yoshinaga K, Saeki H, Morita M, Kakeji Y, Gillespie DA, Yamamoto K, Takata M, Kitao H and Maehara Y: ATR-Chk1 signaling pathway and homologous recombinational repair protect cells from 5fluorouracil cytotoxicity. DNA repair 11: 247-258, 2012.

9 Martino-Echarri E, Henderson BR and Brocardo MG: Targeting the DNA replication checkpoint by pharmacologic inhibition of Chk1 kinase: a strategy to sensitize APC mutant colon cancer cells to 5-fluorouracil chemotherapy. Oncotarget 5: 9889-9900, 2014.
10 Lin PT, Gleeson JG, Corbo JC, Flanagan L and Walsh CA: DCAMKL1 encodes a protein kinase with homology to doublecortin that regulates microtubule polymerization. J Neurosci 20: 9152-9161, 2000.

11 Nakanishi Y, Seno H, Fukuoka A, Ueo T, Yamaga Y, Maruno T, Nakanishi N, Kanda K, Komekado H, Kawada M, Isomura A, Kawada K, Sakai Y, Yanagita M, Kageyama R, Kawaguchi Y, Taketo MM, Yonehara S and Chiba T: Dclk1 distinguishes between tumor and normal stem cells in the intestine. Nat Genet 45: 98-103, 2013.

12 Chandrakesan P, Yao J, Qu D, May R, Weygant N, Ge Y, Ali N, Sureban SM, Gude M, Vega K, Bannerman-Menson E, Xia L, Bronze M, An G and Houchen CW: Dclk1, a tumor stem cell marker, regulates pro-survival signaling and self-renewal of intestinal tumor cells. Mol Cancer 16: 30, 2017.

13 Gao T, Wang M, Xu L, Wen T, Liu J and An G: DCLK1 is upregulated and associated with metastasis and prognosis in colorectal cancer. J Cancer Res Clin Oncol 142: 2131-2140, 2016.

14 Takiyama A, Tanaka T, Kazama S, Nagata H, Kawai K, Hata K, Otani K, Nishikawa T, Sasaki K, Kaneko M, Emoto S, Murono $\mathrm{K}$, Takiyama H and Nozawa H: DCLK1 expression in colorectal polyps increases with the severity of dysplasia. In Vivo 32: 365371, 2018.

15 Weygant N, Qu D, Berry W, May R, Chandrakesan P, Owen DB, Sureban SM, Ali N, Janknecht R and Houchen CW: Small molecule kinase inhibitor LRRK2-IN-1 demonstrates potent activity against colorectal and pancreatic cancer through inhibition of doublecortin-like kinase 1. Mol Cancer 13: 103, 2014.

16 Kawamura D, Takemoto Y, Nishimoto A, Ueno K, Hosoyama T, Shirasawa B, Tanaka T, Kugimiya N, Harada E and Hamano K: Enhancement of cytotoxic effects of gemcitabine by Dclk1 inhibition through suppression of Chk 1 phosphorylation in human pancreatic cancer cells. Oncol Rep 38: 3238-3244, 2017.

17 Ingraham HA, Tseng BY and Goulian M: Nucleotide levels and incorporation of 5-fluorouracil and uracil into DNA of cells treated with 5-fluorodeoxyuridine. Mol Pharmacol 21: 211-216, 1982.

18 Wilkinson DS, Tlsty TD and Hanas RJ: The inhibition of ribosomal RNA synthesis and maturation in Novikoff hepatoma cells by 5-fluorouridine. Cancer Res 35: 3014-3020, 1975.

19 Lu LW, Chiang GH, Tseng WC and Randerath K: Effects of 5fluorouridine on modified nucleosides in mouse liver transfer RNA. Biochem Biophys Res Commun 73: 1075-1082, 1976.

20 Hartmann KU and Heidelberger C: Studies on fluorinated pyrimidines. XIII. Inhibition of thymidylate synthetase. J Biol Chem 236: 3006-3013, 1961.

21 Huang P, Chubb S, Hertel LW, Grindey GB and Plunkett W: Action of 2',2'-difluorodeoxycytidine on DNA synthesis. Cancer Res 51: 6110-6117, 1991.

22 Bouffard DY, Laliberte J and Momparler RL: Kinetic studies on 2',2'-difluorodeoxycytidine (Gemcitabine) with purified human deoxycytidine kinase and cytidine deaminase. Biochem Pharmacol 45: 1857-1861, 1993.

23 Hui YF and Reitz J: Gemcitabine: a cytidine analogue active against solid tumors. Am J Health-System Pharm 54: 162-170, 1997.

Received August 11, 2018

Revised September 19, 2018

Accepted September 28, 2018 